\title{
3-D PATTERNED MICROSTRUCTURES USING INCLINED UV EXPOSURE AND METAL TRANSFER MICROMOLDING
}

\author{
Seong-O Choi ${ }^{1}$, Swaminathan Rajaraman ${ }^{1}$, Yong-Kyu Yoon ${ }^{1}$, Xiaosong $\mathrm{Wu}^{2}$ and Mark. G. Allen ${ }^{1}$ \\ School of Electrical and Computer Engineering ${ }^{1}$, School of Polymer, Textile and Fiber Engineering ${ }^{2}$, \\ Georgia Institute of Technology, \\ Atlanta, GA, 30332, USA
}

\begin{abstract}
We report a fabrication approach for three-dimensional (3-D) microstructures having functional metal patterns by using inclined UV lithography and 3-D metal transfer micromolding. Inclined rotational UV exposure using $\mathrm{SU}-8$ has been exploited to simultaneously generate gradually varying 3-D structures with different heights in a single mask. For 3-D metal patterning, a metal transfer micromolding process has been utilized, where prepatterned metal electrodes are formed on a polydimethylsiloxane (PDMS) mold prior to transfer, and these patterned metal structures are transferred to the molded 3-D microstructures during the micromolding process. Two approaches for metal prepatterning have been utilized: one is selective metal removal by planar pattern transfer on an uneven surface and the other is pattern transfer using a shadow mask. Two test vehicles of 3-D patterned microstructures implemented by this process are demonstrated: an electroporation microneedle array and a 3-D microelectode array (MEA). Insertion tests on pig skin using the fabricated microneedle array with different heights have been successfully performed, showing different penetration depths by fluorescent imaging.
\end{abstract}

\section{INTRODUCTION}

Recent advances in micromolding have enabled rapid progress in the cost-effective fabrication of three-dimensional (3D) microstructures [1]. In general, the micromolding process consists of master structure fabrication, negative mold fabrication, and casting and separation. The fabrication of master structures often relies on advanced UV lithography using SU-8 for its complex 3-D structure capability [2]. As a mold material, PDMS has been widely utilized because of its mechanical compliance and faithful feature reproduction. In the conventional molding process, direct casting of a variety of polymers into the mold is performed to complete the process. In the case of patterning of metals on the three-dimensional surface to functionalize or enable other applications such as microelectrode arrays (MEAs) or an electrically active microneedle array for electroporation, either complex lithographic approaches [3], electrodeposited resists [4] or serial direct laser patterning [5] needs to be applied to the molded 3-D structures.

Alternatively, the 3-D micro electrodes can be formed during the molding process. The so-called 3-D metal transfer micromolding process performs metallization and subsequent patterning on the PDMS mold prior to casting polymer, and these patterned metal structures are transferred to the molded 3-D microstructures during the molding process as shown in Figure 1. This process can be thought of as a three-dimensional extension of 2-D metal transfer processes used in nanoprinting [6], which exploits the difference in surface energy between PDMS and the microstructure material to enable metal transfer.

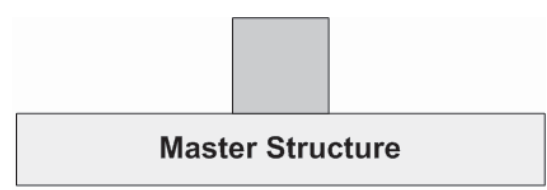

(a)

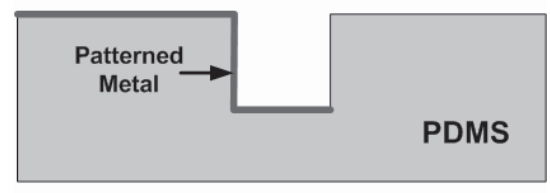

(b)

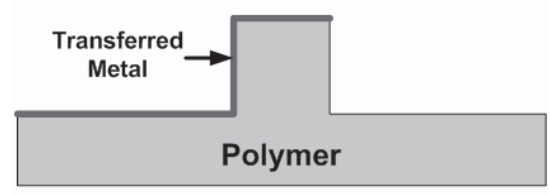

(c)

Figure 1. Concept of 3-D metal transfer micromolding: (a) Fabrication of a master structure, (b) Fabrication of PDMS mold from the master followed by metal deposition and patterning, (c) Formation of 3-D metal-patterned polymeric microstructure.

The process has several advantages. First, since metallization and metal patterning is performed on the mold structure, which is a negative form of the master structure, the process has an additional degree of freedom in 3-D metal patterning for the structures otherwise metal patterning may not be convenient with. Secondly, the pre-deposited or patterned metal layers contribute to enhancing moldability by increasing wettability on the mold surface for liquid polymer casting [7]. Third, the transferred metal does not necessarily require an intermediate adhesion layer as in conventional physical vapor deposition, further simplifying the process.

This paper consists of 3-D microfabrication using inclined UV lithography and micromolding for master structure fabrication, and subsequent 3-D metal transfer molding in which two metal patterning processes are demonstrated. The process is illustrated through two sample structures; one of the structures, an array of microneedles of varying heights, is tested for its insertion capabilities.

\section{3-D MICROSTRUCTURE FABRICATION}

A rigid SU-8 mold is fabricated using inclined UV lithography [8-10] to form negative concave shapes of various depths as shown in Figure 2a, b. Both mask dimensions as well as the incident angle of UV light determine the depth of the mold. This rigid mold is used to produce a mold master (Figure 2c) from 
PDMS or other suitable material (such as $\mathrm{Ni}$ in the case of electroplating). The mold master is then used to create a flexible replica (Figure $2 \mathrm{~d}$ ) of the original rigid mold; this flexible mold should be made from PDMS due to both its low modulus and low surface energy. This flexible mold is then optionally metallized and patterned as described below. Polymer microstructures with various heights are then fabricated from the flexible mold (Figure 2e, f).
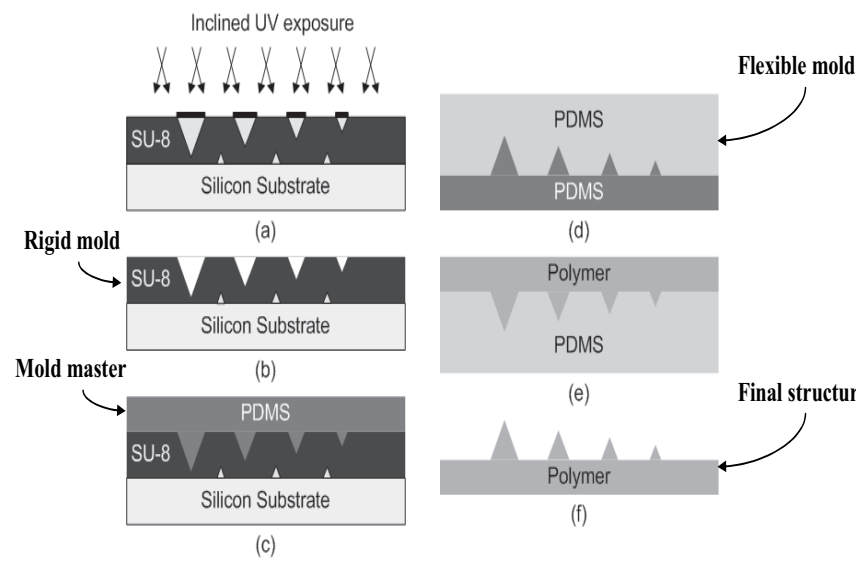

Figure 2. Fabrication steps for 3-D microstructure.

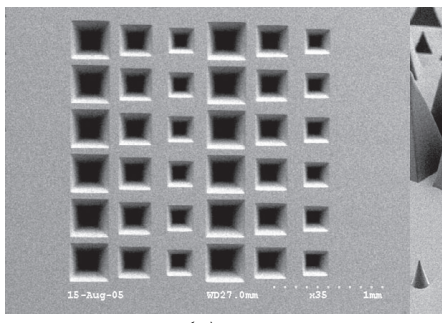

(a)

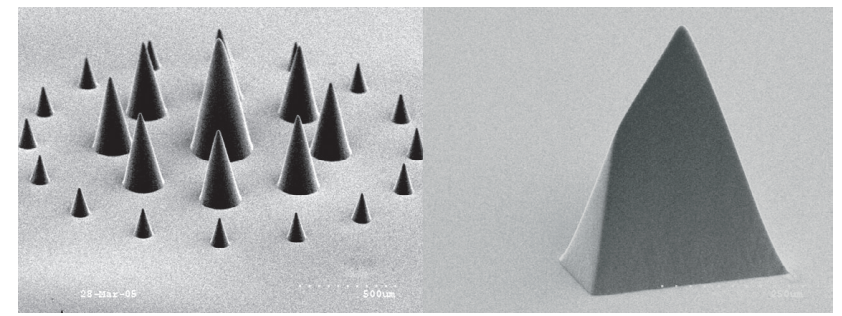

(c)

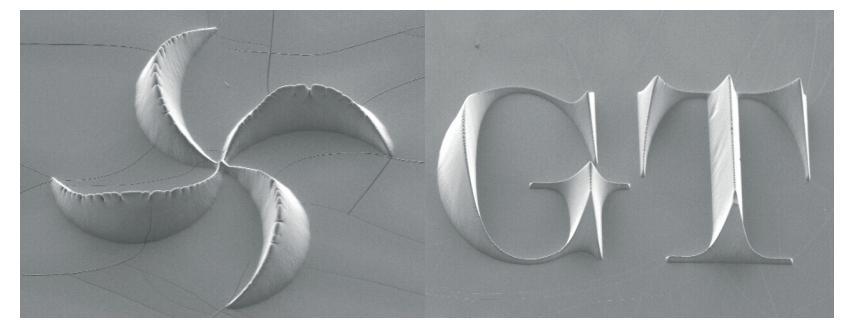

(e)

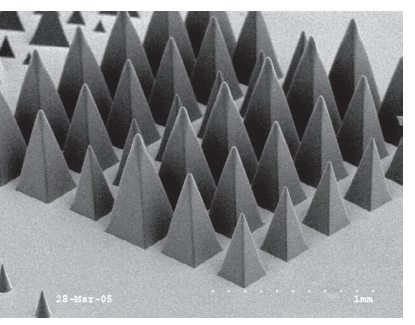

(b)

(d)

$(f)$

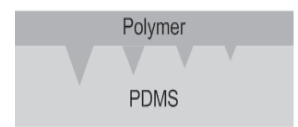

(e)

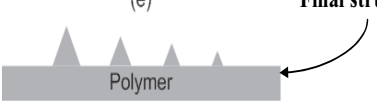

(†)
Figure 3. Fabricated structures: (a) rigid SU-8 mold, (b) variable height microneedle array cast from a flexible mold, (c) cone-shape microneedle, (d) beveled-tip microneedle, (e) vane-shape structure, (f) GT $\log o$
Figure 3 shows various structures fabricated from inclined rotational exposure combined with the subsequent molding process. With differing mask shapes and inclination angles, molded copies of gradually-varying complex 3-D structures have been achieved. One application of interest for this technology is 3-D microneedle array fabrication. Advantages of this process include (1) easy control of the height and the tip angle of the microneedle by control of inclination angle; (2) simultaneous fabrication of microneedles of various heights by controlling the mask footprint; (3) no need for subsequent wet or dry etching for sharp tip fabrication.

\section{3-D METAL TRANSFER MICROMOLDING}

In 3-D metal transfer micromolding, metallization is performed on the negative form of the master made of PDMS. Two metallization schemes and subsequent 3-D metal transfer micromolding have been demonstrated. One scheme is to use selective metal removal from an intentionally formed non-planar mold surface using a 2-D metal transfer process. The other is to use selective metal deposition onto and into mold features using a shadow mask.

High Surface Energy Plate Approach: Figure 4 shows the fabrication steps using a selective metal removal process for an electrically functionalized molded electroporation microneedle array, which requires conductive microneedles and electrical isolations between needle rows. The PDMS mold is fabricated by a two-step SU-8 process and subsequent PDMS molding to form a protruding feature which will ultimately electrically isolate each row $(\mathrm{a}, \mathrm{b})$. After depositing $\mathrm{Au}$ on the mold (c), the Au layer on the protruding structure is removed by bringing a high surface energy plate in contact with the mold (d), transferring the metal on the protruding surface to the plate in a 2-D metal transfer scheme. UV-curable resin is then cast into the mold and optically cured (e). The pre-patterned $\mathrm{Au}$ layer is then transferred during the demolding process (f), resulting in the formation of a polymeric microneedle array with electrical isolations between needle rows (Figure 5). This process avoids the issues associated with conventional metal patterning, since conventional approaches will experience difficulties in patterning of the isolation layer on the bottom of the substrate due to the protruding needle structures.

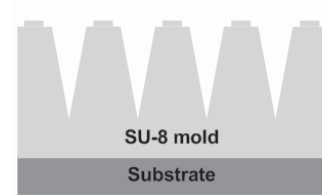

(a)

(b)

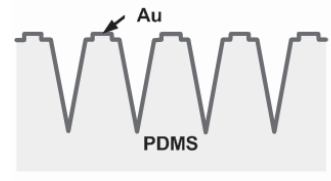

(c)

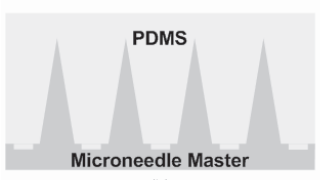

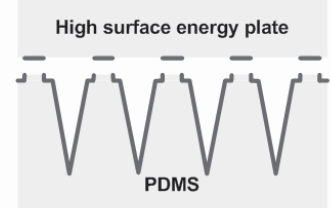

(d)
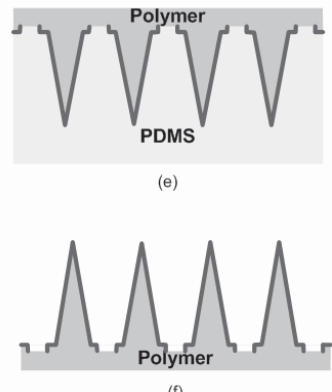

Figure 4. Fabrication steps for metal transfer onto a 3-D structure using a high surface energy plate 


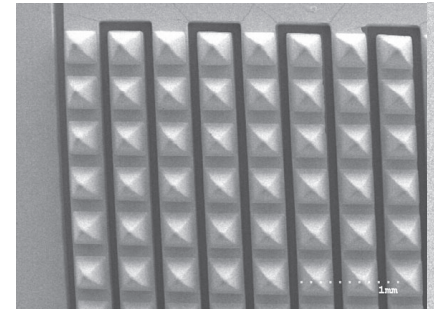

(a)

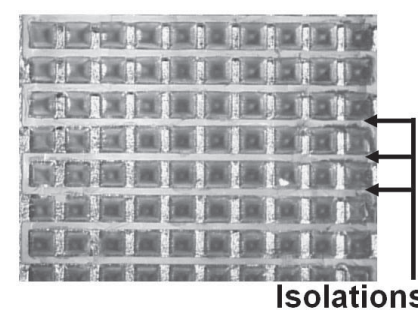

(c)

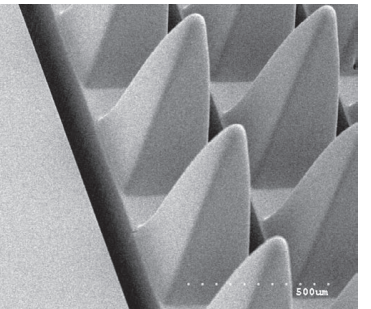

(b)

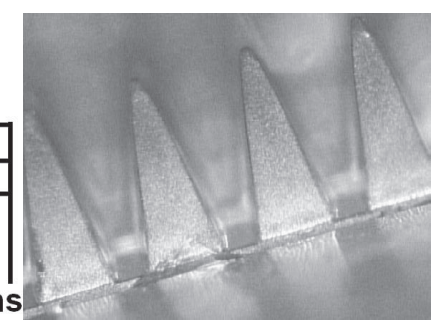

(d)
Figure 5. Fabricated microneedle arrays with applications in electroporation: $(a, b)$ SEM of the mold master, (c,d) Optical photomicrograph of metal patterned structures.

Shadow Mask Approach: Metal can be patterned on the three-dimensional molds using a shadow mask approach. The shadow mask used in this paper was made from $125-150 \mu \mathrm{m}$ thick Kapton sheets using excimer laser ablation. The ablation is achieved at $250 \mathrm{~mJ}$ energy ( $20 \%$ attenuation) at the rate of 50 $60 \mu \mathrm{m}$ per cut. Feature sizes as small as $20 \mu \mathrm{m}$ can be achieved using these parameters. This mask is then aligned with the PDMS mold (refer to section on 3-D Microstructures). Alignment marks are cut on the shadow mask to aid this process. The mask is held in position (proximity contact). This substrate is then loaded into a filament evaporator and $\mathrm{Au} / \mathrm{Cr}(1 \mu \mathrm{m} / 100 \AA)$ is evaporated. We have experimented with $\mathrm{Au} / \mathrm{Cr}$ evaporation instead of just $\mathrm{Au}$, because theoretically the adhesion force between $\mathrm{Cr}$ and cast polymer is higher than that between Au and polymer. However, it turned out that in both cases adhesion between the metal and polymer passes the Scotch tape test. SU-8 is then cast into this mold baked and blanket exposed. The SU-8 tower array is carefully peeled off from the PDMS mold after post-exposure baking. The $\mathrm{Au} / \mathrm{Cr}$ metal layer is transferred from PDMS to SU-8 and becomes $\mathrm{Cr} / \mathrm{Au}$ on the SU-8 structure in the case of both $\mathrm{Au}$ and $\mathrm{Cr}$ being evaporated. Due to the usage of shadow masks the towers have metal lines at various heights on them satisfying the 3D metallization needed for improved MEA performance [11]. Also address lines to contact these electrodes are achieved to the end of the chip. The chip could potentially be mounted and wirebonded for use in MEA experiments. Figure 6 details the process steps and Figure 7 shows optical micrographs of the fabricated structures.

\section{APPLICATIONS AND DISCUSSION}

One application of interest for this technology is an electrically functional 3-D active microneedle array. The negative mold master has been fabricated using inclined rotational UV exposure with SU-8 as described in the previous section. Combined with the 3-D metal transfer molding process, costeffective, mass-producible electrically active 3-D microstructures can be fabricated. These microneedles could be utilized as an electroporation microneedle array for either gene delivery through skin or electrochemotherapy of highly-localized solid tumors.
Other applications of interest include simultaneous local and systemic delivery of drug to blood vessels; drug delivery system through a cornea, which is not flat in nature; simultaneous measurement of biopotentials such as action potential at different sites (3-D MEA); and measurement of electrical properties of skin as a function of depth.

For these active microneedle applications, the strength of the needles and the ability to penetrate tissue has been tested. An array of microneedles with various heights that was made from biodegradable polymer, polylactic acid (PLA) has been used for pig skin in vitro. Red dye was spread on the pig skin, and a microneedle array was inserted with a force of approximately $10 \mathrm{~N}$. After removing the microneedle array, the red dye on the surface was removed with deionized water. Cryosection microscopy was used to examine the cross-section of the pig skin (Figure 8), and showed that the microneedle array was successfully inserted with different penetration depths. The tip diameter of the microneedle array was less than $20 \mu \mathrm{m}$.

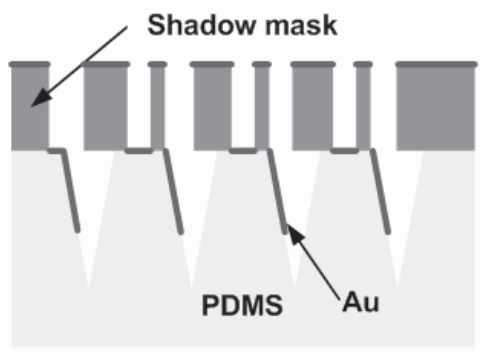

(a)

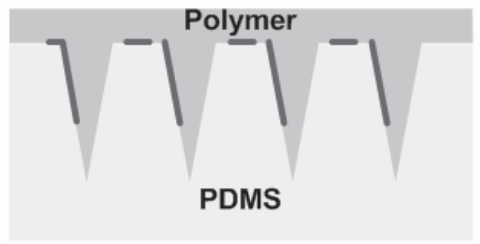

(b)

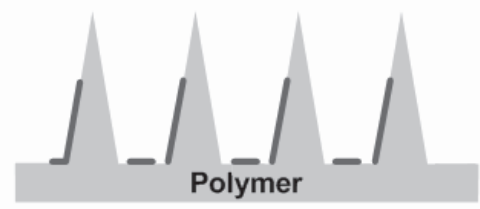

(c)

Figure 6. Fabrication steps for metal transfer onto a 3-D structure using a shadow mask

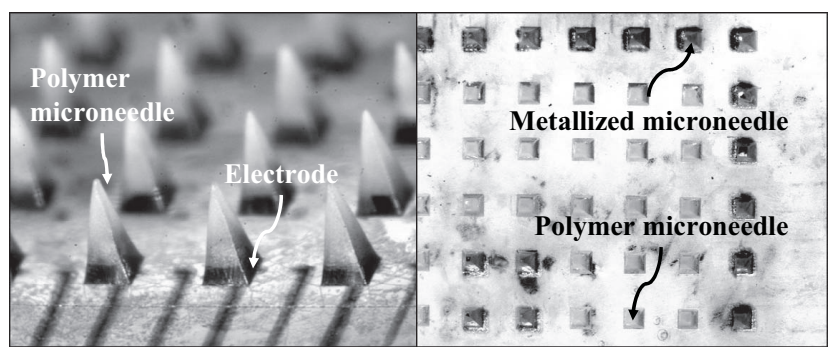

(a)

(b)

Figure 7. Fabricated micro-tower arrays: (a) electrodes with address lines and metal lines at various heights, (b) top view of the selectively metallized micro-towers 


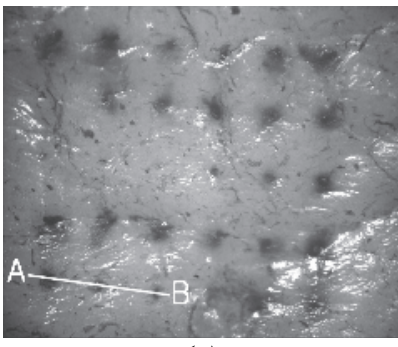

(a)

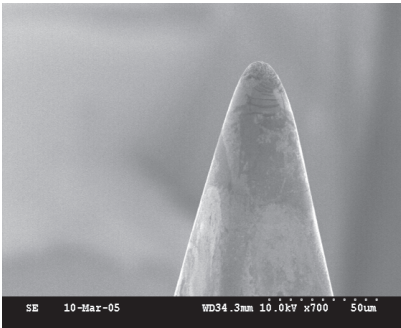

(c)

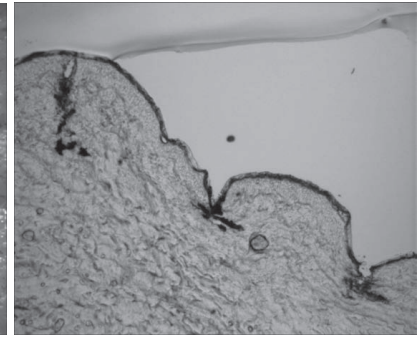

(b)

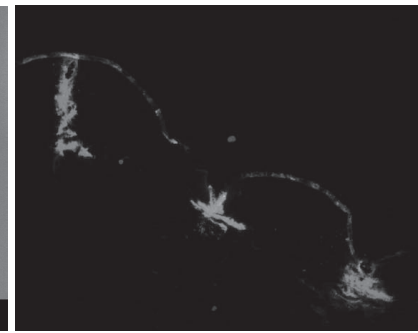

(d)
Figure 8. Optical photomicrograph of pig skin after insertion test with microneedle array of various heights: (a) visible light image (left: plan view; right: cross-section of $A-B$ ), (c) SEM micrograph of microneedle tip (scale bar indicates $50 \mu \mathrm{m}$ ), (d) fluorescent light image of the cross-section showing 3 different penetration depths.

\section{CONCLUSIONS}

We have developed a technology for the fabrication of 3-D microstructures bearing metal patterns by combining inclined UV exposure and metal transfer micromolding. We have demonstrated two separate techniques for selective metal definition on the final structures: one using a high surface energy plate and the other using a shadow mask. In both these techniques metal has been selectively defined on the master mold and then transferred to the final structure during molding. This technology is potentially applicable to fabricate an electrically active microneedle array for either gene therapy or electrochemotherapy, 3-D microelectrode array for neural stimulation/recording, and so on.

\section{ACKNOWLEDGEMENTS}

We wish to acknowledge Mr. Richard H. Shafer for valuable technical discussions. Also we would like to thank Mr. Gary Spinner and the staff of Microelectronics Research Center (MiRC) at Georgia Tech for their efforts in running the cleanroom efficiently. We also appreciate the efforts of Dr. Jung-Hwan Park, Mr. Jungwoo Lee, and Prof. Mark Prausnitz for their assistance with the microneedle insertion tests into pig skin.

\section{REFERENCES}

[1] G. Vozzia, C. Flaimb, A. Ahluwaliaa, and S. Bhatiab, "Fabrication of PLGA scaffolds using soft lithography and microsyringe deposition", Biomaterials, 24 (2003), pp. 2533-2540 [2] Y.-K. Yoon, J.-H. Park, and M. G. Allen, "Muldirectional UV Lithography for Complex 3-D MEMS Structures", J. MEMS (2006), in press

[3] N. P. Pham, E. Boellaard, J. N. Burghartz, and P. M. Sarro, "Photoresist Coating Methods for the Integration of Novel 3-D RF Microstructures", J. MEMS, 13 (2004), pp. 491-499.
[4] S. Linder, H. Baltes, F. Gneadinger and E. Doering, "Photolithograhy in Anisotropically Etched Grooves", Proceedings of the IEEE MEMS Conference (MEMS'96), San Diego, CA, 2/11-15/96, IEEE (1996), pp. 38-43.

[5] Y. Choi, S. Choi, R. H. Shafer, and M. G. Allen, "Highly Inclined Electrodeposited Metal Lines Using an Excimer Laser Patterning Technique", Technical Digest of the $13^{\text {th }}$ International Conference on Solid-state Sensors, Actuators and Microsystems (Transducers'05), Seoul, Korea, 6/6-9/05, IEEE (2005), pp. 14691472.

[6] S. Hur, D. Khang, C. Kocabas, and J. A. Rogers, "Nanotransfer Printing by use of Noncovalent Surface Forces: Applications to Thin-film Transistors that use Single-Walled Carbon Nanotube Networks and Semiconducting Polymers", Appl. Phys. Lett., 85 (2004), pp. 5730-5732

[7] X. Wu, Y. Zhao, Y. K. Yoon, S. O. Choi, J. H. Park, and M. G. Allen, "Wafer-scale Micromolding of Unitary Polymeric Microstructures with Simultaneously Formed Functional Metal Surface", Proceedings of $\mu$ TAS 2005 conference, Boston, MA, 10/09-13/2005, Transducers Research Foundation (2005), pp.205207

[8] M. Han, W. Lee, S.-K. Lee, and S. S. Lee, "3-D Microfabrication using Inclined/Rotated UV Lithography", Sensors and Actuators A, 111 (2004), pp. 14-20.

[9] H. Sato, T. Kakinuma, J. S. Go, and S. Shoji, "In-channel 3-D Micromesh Structures using Maskless Multi-Angle Exposures and their Microfilter Application", Sensors and Actuators A, 111 (2004), pp. 87-92.

[10] Y.-K. Yoon, J.-H. Park, F. Cros, and M. G. Allen, "Integrated Vertical Screen Microfilter System using Inclined SU-8 Structures", Proceedings of the IEEE MEMS Conference (MEMS'03), Kyoto, Japan, 1/19-23/2003, IEEE (2003), pp. 227230.

[11] M.O. Heuschkel, M. Fejtl, M. Raggenbass, D. Bertrand, P. Renaud, "A Three Dimensional Multi-Electrode Array for MultiSite Stimulation and Recording in Acute Brain Slices", $J$. Neuroscience Methods, 114 (2002), pp. 135-148. 